\title{
THE APRIL MEETING OF THE AMERICAN MATHEMATICAL SOCIETY IN NEW YORK.
}

THE one hundred and seventieth regular meeting of the Society was held in New York City on Saturday, April 25, 1914, extending through the usual morning and afternoon sessions. The attendance included the following forty-four members:

Mr. E. S. Allen, Dr. F. W. Beal, Mr. R. D. Beetle, Professor W. J. Berry, Professor E. G. Bill, Professor G. D. Birkhoff, Professor Maxime Bôcher, Professor Joseph Bowden, Professor B. H. Camp, Professor F. N. Cole, Dr. G. M. Conwell, Professor J. L. Coolidge, Professor Elizabeth B. Cowley, Professor L. P. Eisenhart, Dr. C. A. Fischer, Professor T. S. Fiske, Professor W. B. Fite, Dr. G. M. Green, Dr. T. H. Gronwall, Professor C. N. Haskins, Professor H. E. Hawkes, Mr. S. A. Joffe, Professor Edward Kasner, Dr. J. K. Lamond, Professor W. R. Longley, Professor James Maclay, Dr. E. J. Miles, Mr. B. E. Mitchell, Dr. H. H. Mitchell, Professor C. L. E. Moore, Dr. R. L. Moore, Dr. F. M. Morgan, Professor Alexander Pell, Dr. Anna J. Pell, Dr. H. W. Reddick, Professor R. G. D. Richardson, Dr. J. E. Rowe, Dr. Clara E. Smith, Professor D. E. Smith, Professor P. F. Smith, Mr. J. M. Stetson, Professor H. W. Tyler, Mr. H. S. Vandiver, Mr. H. E. Webb.

Ex-President Maxime Bôcher occupied the chair, being relieved by Vice-President L. P. Eisenhart, Ex-President T. S. Fiske, and Professor H. W. Tyler. The Council announced the election of the following persons to membership in the Society: Dr. T. H. Brown, Yale University; Dr. Josephine E. Burns, University of Illinois; Professor C. F. Gummer, Queen's University, Kingston, Ontario; Mr. G. M. Hayes, College of the City of New York; Dr. W. L. Miser, University of Minnesota; Professor Maximilian Philip, College of the City of New York; Mr. S. A. Schwarz, College of the City of New York; Professor A. G. Smith, State University of Iowa; Mr. R. A. Wetzel, College of the City of New York. Ten applications for membership in the Society were received.

Professor L. E. Dickson was reelected as member of the 
editorial committee of the Transactions for a term of three years beginning October, 1914. It was decided to hold the annual meeting of the Society this year in New York City.

Fifteen members gathered at the usual dinner in the evening.

The publication was announced of the Madison Colloquium Lectures of Professors L. E. Dickson and W. F. Osgood. The book has just appeared as volume 4 of the series of Colloquium Lectures.

The following papers were read at this meeting:

(1) Mr. A. R. Schwertzer: "An extension of functional equations."

(2) Professor L. P. Eisenhart: "Transformations of conjugate systems with equal point invariants."

(3) Dr. H. H. Mitchell: "The subgroups of the quaternary abelian linear group."

(4) Mr. R. D. BeEtLe: “On the complete independence of Schimmack's postulates for the arithmetic mean."

(5) Mr. H. S. Vandiver: "Extension of the criteria of Wieferich and Mirimanoff in connection with Fermat's last theorem."

(6) Professor E. G. BILL: "Note on the curvature of a regular curve in non-euclidean space."

(7) Mr. S. A. JofFE: "Triangles whose sides are three consecutive integers and whose area is an integer."

(8) Professor G. D. Birkhoff: "The restricted problem of three bodies. Second paper."

(9) Professor A. B. CoBlE: "Cremona groups determined by point sets."

(10) Dr. Anna J. Pell: "Non-homogeneous linear equations in infinitely many unknowns."

(11) Miss L. D. Cummings: "On a method of comparison for triple systems."

(12) Professor C. L. E. Moone: "A geometry whose element of arc is a linear differential form."

(13) Professor C. L. E. Moore: "On the centers and radii of curvature of curves traced on a surface in an $n$-space."

(14) Dr. J. E. Rowe: "Invariants of the rational plane" quintic and of rational curves of odd order."

(15) Dr. J. K. LAMOND: "Some applications of a theorem of W. H. Young."

(16) Professor Maxime Bôcher: "On a small variation which renders a linear differential system incompatible." 
(17) Professor R. C. Archibald: "Euclid's book on divisions of figures."

(18) Mr. C. E. Wilder: "On the degree of approximation to discontinuous functions by trigonometric sums. Second paper."

(19) Dr. E. J. MrLes: “Transversality and orthogonality of space extremals."

(20) Mr. P. R. Rider: "Broken extremals in space."

(21) Dr. R. L. Moone: "Linear order in terms of point and limit."

(22) Dr. T. H. Gronwall: "Triply orthogonal systems containing one family of minimal surfaces."

Mr. Wilder's paper was communicated to the Society by Dr. Dunham Jackson. Mr. Rider was introduced by Dr. Miles. The papers of Mr. Schweitzer, Professor Coble, Miss Cummings, Professor Bôcher, Professor Archibald, Mr. Wilder, and Dr. Miles were read by title. Abstracts of the papers follow below. The abstracts are numbered to correspond to the titles in the list above.

1. Mr. Schweitzer's paper is concerned with the determination of the properties of the functions satisfying the following functional equations:

$$
\begin{aligned}
\psi\{\theta(x) \phi(y)-\theta(y) \phi(x)\} & =\sqrt{\psi \theta(x+y)} \sqrt{\psi \theta(x-y)}, \\
\psi_{1}\left\{\theta_{1}(x) \theta_{1}(y)-\phi_{1}(x) \phi_{1}(y)\right\} & =\sqrt{\psi_{1} \theta_{1}(x+y)} \sqrt{\psi_{1} \theta_{1}(x-y)} .
\end{aligned}
$$

By putting $\phi(x)=1-\theta(x)$ and $\phi_{1}(x)=1-\theta_{1}(x)$, one obtains functional equations previously discussed by the author.

2. When the cartesian coordinates of a surface $S$ are solutions of an equation of the form

$$
\frac{\partial^{2} \theta}{\partial u \partial v}+\frac{\partial \rho}{\partial v} \frac{\partial \theta}{\partial u}+\frac{\partial \rho}{\partial u} \frac{\partial \theta}{\partial v}=0
$$

the parametric conjugate system is said to have equal point invariants. Professor Eisenhart shows that when another solution of this equation is known which is linearly independent of the coordinates of $S$, the coordinates of a second surface $S_{1}$, referred to a similar conjugate system, can be 
found by quadratures, the surfaee $S_{1}$ being such that for the congruence of lines joining corresponding points on $S$ and $S_{1}$ the developables meet the latter in the parametric conjugate systems. This result is established by an adaptation of an important theorem due to Moutard. This transformation of $S$ into $S_{1}$ is called a transformation $K$. If two transforms $S_{1}$ and $S_{2}$ of a surface $S$ are known, a one-parameter family of surfaces $S^{\prime}$ can be found by quadratures such that each surface $S^{\prime}$ is in the relation of a transformation $K$ with $S_{1}$ and of a different transformation $K$ with $S_{2}$; moreover, the corresponding points $M, M_{1}, M_{2}, M^{\prime}$ on four surfaces $S, S_{1}$, $S_{2}, S^{\prime}$ lie in a plane $\pi$, and all the corresponding points $M^{\prime}$ for surfaces of the family lie on a line through $M$. This line and the line $M_{1} M_{2}$ meet in the point of contact of the plane $\pi$ with its envelope. These results are equally true in $n$-space of reseaux and congruences in the sense adopted by Guichard. In 3-space a transformation $K$ gives rise to four $W$-congruences in the relation of a theorem of permutability as considered by Bianchi, and conversely four such $W$-congruences determine four pairs of surfaces in the relations of transformations $K$. The transformations $D_{m}$ of isothermic surfaces, studied by Darboux and Bianchi, are the only transformations $K$ for which the two surfaces envelop a two-parameter family of spheres. The equations of the theorem of permutability of the transformations $D_{m}$ are an immediate consequence of the general equations for transformations $K$. The results of this paper reveal the fundamental rôle played by the theorem of Moutard in the recent theory of transformations of surfaces.

3. One of the most interesting of the modular groups is the abelian linear group. For the Galois field $G F(p)$ it has been investigated by Jordan, and for the general Galois field $G F\left(p^{n}\right)$ by Dickson. For the case of four variables considerable work has been done on the determination of its subgroups, but the problem seems to have been completely solved only for $n=1, p=2,3$. Dickson however has proved that for $n=1, p>3$ there is no subgroup of index less than $p^{3}+p^{2}+p+1$. Dr. Mitchell determines the maximal subgroups in case $p$ is an odd prime and $n$ any integer. He finds that except for $n=1, p=3$ there is no subgroup of index less than $p^{3 n}+p^{2 n}+p^{n}+1$. 
4. In volume 68 of the Mathematische Annalen, Schimmack gave a set of axioms for the arithmetic mean, and showed that these axioms were independent in the usual sense. Mr. Beetle proves that Schimmack's axioms form an interesting example of a completely independent* set.

5. If

$$
x^{p}+y^{p}+z^{p}=0
$$

is satisfied in rational integers prime to each other and to the odd prime $p, \mathrm{Mr}$. Vandiver shows that

$$
\prod_{i=1}^{m-1}\left(\alpha^{2 i}-t\right) \sum_{i=1}^{m-1} \frac{\left(1-\alpha^{2 i}\right) f_{p-1}\left(\alpha^{i}\right)}{\left(1-\alpha^{i p}\right)\left(\alpha^{2 i}-t\right)} \equiv 0(\bmod p),
$$

where $\alpha=e^{\frac{2 \pi \sqrt{-1}}{m}}(m=2,3, \cdots, p-1)$,

$$
f_{k}(a)=\sum_{r=1}^{p-1} r^{k-1} a^{r},
$$

and $t$ is any of the ratios

$$
-\frac{x}{y},-\frac{y}{x},-\frac{x}{z},-\frac{z}{x},-\frac{y}{z},-\frac{z}{y} .
$$

Letting $m=5$ and comparing the resulting congruence with a condition given by Mirimanoff, it follows that $5^{p-1} \equiv 1$ $\left(\bmod p^{2}\right)$ is a criterion for the solution of (1) in rational integers prime to each other and to $p$.

6. In the two ordinary non-euclidean spaces, two tangents to a regular curve $C$ at the points with parameter values $s$ and $s+\Delta s$ form four angles $\Delta_{i} \theta(i=1,2,3,4)$. Of the four limiting values $\lim _{\Delta s=0} \Delta_{i} \theta / \Delta s$, two are identically zero and two are numerically equal and not identically zero. Professor Bill defines the curvature of $C$ at $s$ to be one of these limiting values which is not identically zero.

7. Assuming the three sides of a triangle, $a=2 n-1$, $b=2 n$, and $c=2 n+1$, so that the area is

$$
F=n \sqrt{3(n+1)(n-1)},
$$

* See E. H. Moore: "Introduction to a form of general analysis," New Haven Mathematical Colloquium, Yale University Press, 1910, p. 82. 
Mr. Joffe shows that all the triangles whose sides are three consecutive integers and whose areas are integers can be obtained from the integral solutions of two indeterminate quadratic equations: $y^{2}=3 x^{2}-2$ and $y^{\prime 2}=3 x^{\prime 2}+1$. He then proves that all the integral roots, $x, y$, and $x^{\prime}, y^{\prime}$, of these quadratic equations may be obtained simultaneously by successive addition, and exhibits the results in a table: the proof being based upon two fundamental identities $\Delta x_{i}=2 \Sigma x_{i}$ and $y_{i}=2 x_{i}-x_{i-1}$, and corresponding identities for $x_{i}{ }^{\prime}$ and $y_{i}{ }^{\prime}$.

The sides $b_{i}$ and $b_{i}{ }^{\prime}$ are computed (in two tables) from the formulas $b_{i}=2\left(y_{i}{ }^{2}+1\right)$ and $b_{i}{ }^{\prime}=2\left(2 y_{i}{ }^{2}-1\right)$, and it develops that the following relation holds between three consecutive $b$ 's:

$$
b_{i}-14 b_{i-1}+b_{i-2}=0,
$$

and that exactly the same relation exists in the $b^{\prime \prime}$ 's; furthermore, that $b_{i}=2 y_{2 i-1}^{\prime}$, and $b_{i}^{\prime}=2 y_{2 i}^{\prime}$. The $b^{\prime}$ 's and $b^{\prime \prime}$ s are connected with each other by the formula $b_{i}{ }^{2}-2=b_{2 i-1}^{\prime}$, while $b_{i}{ }^{2}-2=b_{2 i}^{\prime}$.

8. Levi-Civita* has given a notable form of the equations of motion in the restricted problem of three bodies which is free of singularity at collision of the "infinitesimal body" with one of the other two bodies. The paper by Professor Birkhoff contains a new form of the equations of motion free of singularity at collision with either of the two bodies, and thus throughout the entire motion. Interesting applications of this result are possible.

A rigorous demonstration of the existence of retrograde periodic orbits, so long as the motion is confined to an oval about one of the bodies, is obtained. Up to the present time all proofs of existence of periodic orbits have dealt exclusively with the case in which one of the bodies is sufficiently small or the constant of Jacobi is sufficiently large. F. R. Moulton has called attention to the superior simplicity of the retrograde orbits and has made interesting conjectures as to their character. $\dagger$

The very difficult questions connected with the direct

* Acta Mathematiea, vol. 30 (1906), pp. 305-327.

$\dagger$ Proceedings of the Fifth International Congress of Mathematicians, vol. 2, pp. 182-187. 
orbits are considered by Professor Birkhoff, but equally definite results are not obtained.

This paper will appear incorporated with a paper on the same subject presented to the Society at New York at the last annual meeting.

9. In Professor Coble's paper the definition of an invariant of a binary form of order $n$ in terms of the coordinates of the corresponding $n$ points in an $S_{1}$ is generalized to apply to a set $P_{n}{ }^{(k)}$ of $n$ points in an $S_{k}$. It appears that a set $P_{n}{ }^{(k)}$ is associated with a set $P_{n}^{(n-k-2)}$ in such a way that either set projectively defines the other and that corresponding invariants are proportional. If the projectively distinct sets $P_{n}{ }^{(k)}$ or their associated sets be mapped upon the points of a space $\Sigma_{k(n-k-2)}$, the permutations of the points within a set determine in $\Sigma$ a Cremona $G_{n !}{ }^{(k)}$. The groups thus determined by $P_{n}{ }^{(1)}$ or $P_{n}{ }^{(n-3)}$ are the cross-ratio groups of Moore. Those determined by $P_{n}^{(r+1)}$ or $P_{n}^{(n-3-r)}, r>0$, are of additional interest due to the fact that they are subgroups of more comprehensive Cremona groups. For example $P_{6}{ }^{(2)}$ determines in $\Sigma_{4}$ a Cremona $G_{51840}$ isomorphic with the group of the lines on a cubic surface; $P_{7}^{(2)}$ or $P_{7}{ }^{(3)}$ determines in $\Sigma_{6}$ a Cremona $G_{7 ! \cdot 288}$ isomorphic with that of the bitangents of a plane quartic; and $P_{8}{ }^{(2)}$ or $P_{8}{ }^{(4)}$ determines in $\Sigma_{8}$ a Cremona group isomorphic with that of the tritangent planes of a space sextic of genus 4 on a quadric cone, i. e., a sextic for which an even theta vanishes for the zero argument. In all these cases the group is in direct algebraic relation with the corresponding geometric configuration. The extended Cremona groups determined by sets of points other than those mentioned are of infinite order.

10. Using the property of a limited bilinear form that its value can be obtained by summation by rows or by columns, Mrs. Pell shows that the system of non-homogeneous linear equations

$$
\sum_{k=i}^{\infty} a_{i k} x_{k}=c_{i} \quad(i=1,2, \cdots, \infty),
$$

$a_{i i} \neq 0$ being the only restriction on the coefficients, has a solution $\left\{\gamma_{i}\right\}$ such that $\left\{\lambda_{i} \gamma_{i}\right\}$ is of finite norm, for every $\left\{c_{i}\right\}$ such that $\left\{\mu_{i} c_{i}\right\}$ is of finite norm. The constants $\left\{\lambda_{i}\right\}$ 
and $\left\{\mu_{i}\right\}$ are directly and simply expressed in terms of the coefficients $a_{i k}$. Extension is made to systems of linear equations reducible to this type.

11. In Miss Cummings's paper three points fundamental in the study of triple-systems are considered: $(a)$ A method of comparison applicable to triple-systems of any number of elements; (b) an easy process for constructing non-congruent systems; (c) a direct means for determining the sets of transitive elements and the group of a system. That the group does not form an abstract mark for systems containing more than 13 elements is established, and several examples are shown of non-congruent systems with the same group. In this paper a system is characterized by its indices. Two systems with different sets of indices are shown to be distinct, whether their groups are different or identical. Of the 24 systems discussed at least 12 are apparently new. The known systems of Kirkman, Reiss, Netto, Heffter, and E. H. Moore are examined; and the identity or non-congruency of these systems is exhibited. The groups are determined for the 24 systems.

12. It is evident that there are but two kinds of geometries here possible, viz., (1) arc length an exact differential, (2) arc length not an exact differential. The geometries developed by Phillips and Moore* are examples of each kind. Therefore any geometry whose arc length is linear can be mapped (arc length being preserved) on one or the other of these. Using this map, Professor Moore discusses the geometry on a minimum developable surface.

13. Making use of the well known theorems that the osculating planes of all curves traced on a surface through a point $P$ in a fixed direction $t$ generate a 3 -space, and that the locus of this 3-space as $t$ varies is a quadric cone of four dimensions situated in a 5-space, Professor Moore discusses the properties of radii of curvature and of centers of curvature of curves traced through a fixed point on a surface in $S_{n}$.

14. In his dissertation (Transactions, July, 1911) Dr. Rowe has shown how to interpret certain combinants of two binary

* "An algebra of plane projective geometry," Proceedings Amer. Academy of Arts and Sciences, vol. 47 (1912). 
quartics (explicitly written out in Salmon's Higher Algebra) as invariants of the rational plane quartic curve. In this paper he shows how these same combinants may be interpreted as invariants of any rational curve of odd order by means of substitutions which are easily derived. This leads to the development of some interesting facts in regard to rational curves. The rational plane quintic is of especial interest because of its self-conjugacy, and the substitutions which lead to eight of its invariants are actually carried out to form a basis of work in the invariant theory of the rational quintic and to illustrate the general process.

15. In a paper, "On the general theory of integration," published in the Philosophical Transactions in 1905, W. H. Young stated and proved a theorem which expressed the Lebesgue integral of an upper semi-continuous function as the Riemann integral of a monotone decreasing function over an interval. In a previous paper Dr. Lamond stated a generalization of the above theorem and used it to obtain sufficient conditions for the existence and equality of the multiple and iterated $L$-integrals of a function over a field, which was not, necessarily, measurable. From his generalization of Young's theorem Dr. Lamond has now shown that the above sufficient conditions are also, in general, necessary, and has also applied this generalization to the problem of the continuity of an $L$-integral with respect to a parameter.

16. If the homogeneous linear differential equation of the $n$th order, $L(u)=0$, has $k$ linearly independent solutions which satisfy a certain system of homogeneous linear boundary conditions, $U_{i}(u)=0 \quad(i=1,2, \cdots, n)$, the main result proved in Professor Bôcher's paper states that, however small the positive constant $\epsilon$ may be, a continuous function $g(x)$ exists such that $0<g(x)<\epsilon$ and such that the differential equation $L(u)=g(x) u$ has no solution except zero which satisfies the boundary conditions in question.

17. Euclid was the author of not less than nine works. We have approximately complete texts, all carefully edited, of four of these: (1) the Elements, (2) the Data, (3) the Optics, (4) the Phaenomena. In the case of (5) the Pseudaria, (6) the Surface Loci, (7) the Conics, our fragmentary knowl- 
edge, derived wholly from Greek sources, makes conjecture as to their content of the vaguest nature. On (8) the Porisms, Pappus gives extended comment. To (9) the Book on Divisions of Figures, Proclus alone among Greeks makes slight explanatory reference. But in an Arab MS. translated by Woepcke into French (Journal Asiatique, 1851) we have not only the enunciation of all 36 of the propositions of the Book, but also the proofs of four of them. Professor Archibald has endeavored to restore the remaining proofs in euclidean manner, to edit the whole with suitable commentary and historical introduction, which, among other things, takes due account of various Dee and Cotton MSS. attributed to Muhammed Bagdedinus as author. The restoration which is based on Leonardo's Geometria Practicæ (written in 1220 and published by Boncompagni in 1862) will soon be published in England, in book form.

18. This paper is a continuation of the one presented to the Society by Mr. Wilder in February, 1913. It contains several theorems of a more general character than those of the previous paper. The following are typical:

Corresponding to any finite integrable function $f(x)$, of period $2 \pi$, there is for every positive integral value of $n$ a trigonometric sum of order $n$ at most which approximates to $f(x)$ at any point where its derived numbers are all finite, with an error not exceeding $1 / n$ multiplied by a quantity independent of $n$.

Any finite integrable function $f(x, y)$, of period $2 \pi$ in each variable, can be approximated to by an arithmetic mean of partial sums of its Fourier's expansion to terms of order $m-1$ in $x$ and $n-1$ in $y$, at any point having a neighborhood of radius $\delta<\pi$ in which $f(x, y)$ satisfies the Lipschitz condition

$$
\left|f\left(x_{2}, y_{2}\right)-f\left(x_{1}, y_{1}\right)\right| \leqq \lambda \sqrt{\left(x_{2}-x_{1}\right)^{2}+\left(y_{2}-y_{1}\right)^{2}},
$$

with an error not exceeding

$$
\left(\frac{\log m}{m}+\frac{\log n}{n}\right)\left(c_{1} \lambda+c_{2} \frac{\nu}{\delta^{2}}\right),
$$

where $c_{1}$ and $c_{2}$ are absolute constants, and $\nu$ is the difference between the upper and lower limits of $f(x, y)$.

Some of the results can be interpreted so as to give infor- 
mation concerning the accuracy of approximation by means of polynomials.

19. In Dr. Miles's paper the general form of the integrand function is first obtained when the extremals which are transversal to a given surface are also perpendicular to that surface. Then a general relation between the direction of the extremal and the transversal surface is assumed and conditions given under which the integrand function can be determined.

20. Caratheodory and Bolza have treated the case of broken extremals in the plane. Mr. Rider's paper considers the case of broken extremals in space. The Weierstrass-Erdmann corner-condition is derived, and a corner-surface obtained. The analogue of Caratheodory's $\Omega$-function is then found, and the Jacobi necessary condition for an extremum derived, as are also sufficient conditions.

21. Dr. Moore adds the following four axioms to a set of three fundamental axioms formulated by F. Riesz:* and thereby secures a set of seven independent axioms, in terms of point and limit, which is a sufficient basis for a definition of a linear order satisfying Hilbert's axioms of group II. $\dagger$

In the following, $S$ denotes the set of all points under consideration. A set of points is said to be connected if, however it be divided into two complementary subsets, one of them contains a limit point of the other.

Axiom 1. $S$ is connected.

Axiom 2. If $P$ is a point of $S$, then $S-P$ is composed of two connected subsets neither of which contains a limit point of the other. $\ddagger$

Axiom 3. There do not exist three rays such that no two of them have a point in common.

Axiom 4. There exist at least two points.

22. In this paper, Professor Gronwall shows that there exist no triply orthogonal systems containing one family of minimal surfaces, except those already known, where the minimal surfaces are either planes or catenoids of revolution.

F. N. Cole, Secretary.

* Rome Congress, 1908.

$\dagger$ Hilbert, The Foundations of Geometry, Townsend's translation.

$\ddagger$ Such subsets are called rays. 